\title{
SpyGlass DS-guided lithotripsy for pancreatic duct stones in symptomatic treatment-refractory chronic calcifying pancreatitis
}

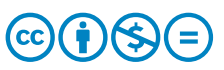

\begin{abstract}
Authors Erwin-Jan M. van Geenen², Torsten Beyna'

Institutions

1 Department of Gastroenterology, Evangelisches Krankenhaus Düsseldorf, Düsseldorf, Germany

2 Department of Gastroenterology and Hepatology, Radboud University Medical Center, Nijmegen, The Netherlands
\end{abstract}

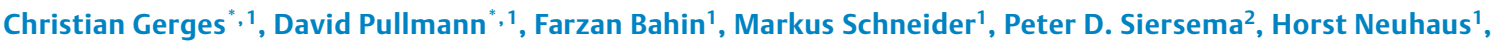

submitted 27.8.2018

accepted after revision 10.10 .2018

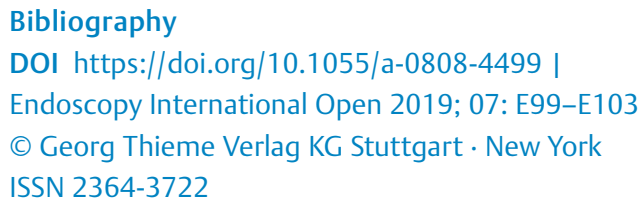

Corresponding author

Christian Gerges, MD, Evangelisches Krankenhaus

Düsseldorf, Gastroenterologie, Kirchfeldstr. 40, 40219

Duesseldorf

Fax: +00492119193960

gerges.christian@gmail.com

\section{ABSTRACT}

Background and study aims Endoscopic retrograde cholangiopancreatography (ERCP) and/or extracorporeal shock wave lithotripsy are first-line therapies for draining an obstructed pancreatic duct (PD) in painful chronic calcifying pancreatitis (CCP). Pancreaticoscopy has shown promising success rates in small series.

Materials and methods This study was a retrospective analysis of a clinical database. Included were all digital single-operator digital video (SOV) pancreaticoscopy-guided interventions $(n=23)$ on CCP patients $(n=20)$ between 2015 and 2017. Success and complication rates were collected from the database. Clinical success was determined by assessing pain level score (NRS) and quality of life (QoL) using standardized questionnaires.

Results Overall technical success rate (successful SOVpancreaticoscopy and PD drainage) was $95 \%$. Adverse events occurred in 7 of 23 procedures (30\%) and included extravasation from the PD $(n=1)$, self-limiting post-sphincterotomy bleeding $(n=1)$ and post-ERCP pancreatitis (PEP) $(n=6)$. At 3- to 6 -month follow-up, $95 \%$ of patients reported improvement in symptoms and reduction in intake of analgesics. Mean NRS decreased from $5.4( \pm 1.6)$ to 2.8 $( \pm 1.8)(P<0.01)$. Clinical success was achieved in $95 \%$ of patients.

Conclusions Digital SOV-guided lithotripsy was found to be safe and effective in this highly selected population of CCP patients. PD decompression had a beneficial effect on pain reduction and QoL.

\section{Introduction}

Endoscopic management for pain from chronic calcifying pancreatitis (CCP) can be achieved by draining an obstructed pancreatic duct (PD) and/or removing obstructing PD calculi [1]. Current guidelines recommend extracorporeal shock wave lithotripsy (ESWL) as first-line treatment of PD stones. However, its limitations include lack of availability and the need for multi-

\footnotetext{
* These authors contributed equally
}

ple ESLW and endoscopic retrograde cholangiopancreatography (ERCP) sessions [2]. The role of pancreatoscopy in PD stone treatment is unclear, with reported success rates between $43 \%$ and $100 \%$, based on small retrospective studies [2 - 7]. In 2015, single-operator digital video cholangiopancreaticoscopy (SOVP) (SpyGlassDS, Boston-Scientific) was introduced. There is limited data on the role of SOVP in the treatment of PD stones in CP. Our aim was to investigate the role of digital SOVP-guided PD stone lithotripsy in patients with CP with regard to safety, technical and short-term clinical success. 

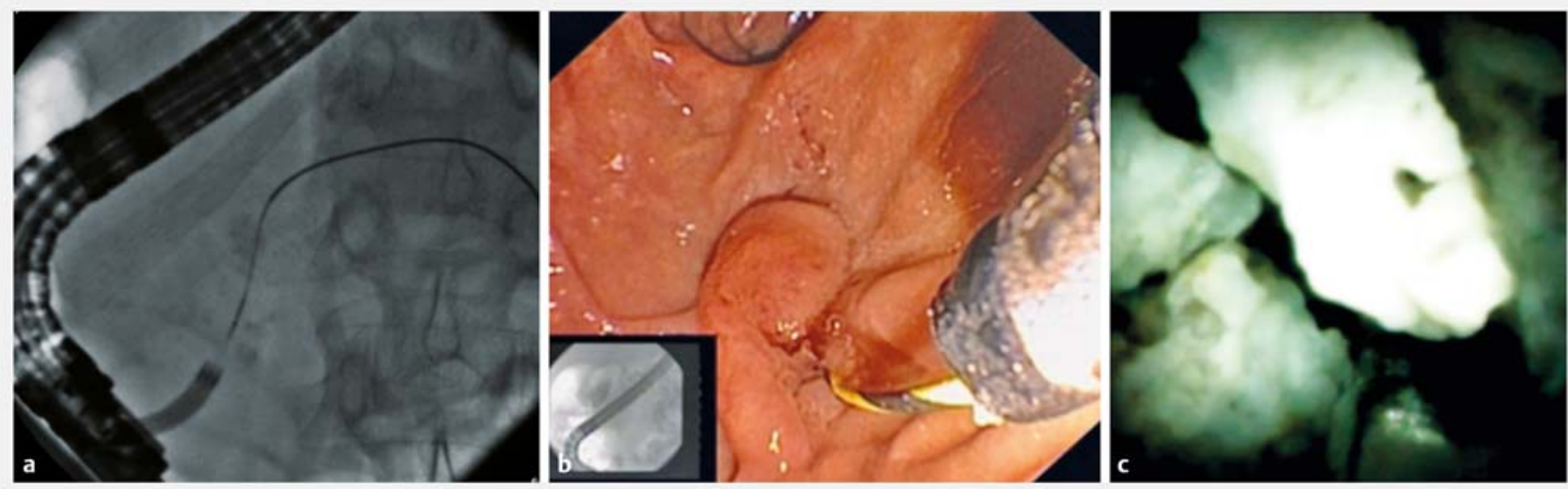

- Fig. 1 a Radiopaque stone in the main pancreatic duct (MPD), b wire-guided cannulation of MPD with SpyGlassDS catheter (Boston Scientific, Natick, Mass, US) after pancreatic sphincterotomy, c direct visualization of the impacted stone in the pancreatic head and Electrohydraulic lithotripsy of the large stone.

\section{Material and methods}

Between 2015 and 2017, all SOVP-guided lithotripsies at two tertiary referral centers were retrospectively identified and analyzed. Inclusion criteria for SOVP were imaging-proven PD stones with upstream dilatation and pain attributable to CCP. Patients with prior unsuccessful ERCP or ESWL were included, as well as patients with asymptomatic pseudocysts and patients with prior pancreatic surgery. A consensus decision to proceed with SOVP was made by an interdisciplinary pancreatic board at each center in shared decision with the patient. All patients meeting the inclusion criteria were consecutively and systematically included in this retrospective study.

Exclusion criteria included age less than 18, pregnancy, abdominal pain not attributable to CCP or unsuitability to receive sedation. All patients had endoscopic ultrasound and abdominal computed tomography and/or magnetic resonance cholangiopancreatography prior to SOVP ( $\triangleright$ Fig. 1). Other treatment options (surgery, ESWL or ERCP) had already been attempted, were deemed less suitable than SOVP or were refused by the patient.

Conventional ERCP techniques such as balloon extraction, mechanical lithotripsy, and balloon dilation of downstream strictures were already used to facilitate stone removal and stricture dilation prior to attempting SOVP in the same session. Pancreatic sphincterotomy preceded SOVP in all cases (either during previous or same intervention); and further treatment of a downstream stricture (between papilla and stone) was performed at the endoscopist's discretion using a 4-mm dilatation balloon (Hurricane RX, Boston Scientific, Nattick, Massachusetts, United States). SOVP was performed using 10.5F SpyglassDS, which was advanced over a .035-inch guidewire (Jagwire, Boston-Scientific) or freehand. Lithotripsy was performed using a Holmium:YAG-laser (AurigaXL, StarMedTech) with a 270- $\mu \mathrm{m}$ optical fiber $(8 \mathrm{~Hz}, 2400 \mathrm{~mJ})$ or electrohydraulic lithotripsy (EHL) utilizing either a Lithotron EL27 generator with a 2.4F probe (Walz Elektronik, Rohrdorf, Germany) or an Autolith tough biliary EHL system with 1.9 F probe (Northgate Technol-
ogies/Boston Scientific, Nattick, Massachusetts, United States) with high power and maximum pulses (30) at the endoscopist's discretion. Intermittent PD irrigation with saline facilitated target stone visualization and lithotripsy ( $\triangleright$ Fig. 1 ).

After lithotripsy, fragments were extracted using balloons or baskets followed by pancreatic plastic stent placement (510 Fr.) for prophylaxis against post-ERCP pancreatitis (PEP) and/or PD decompression. The type/length of the PD stent was based on the diameter, length and position of the stricture. Prophylactic rectal nonsteroidal anti-inflammatory drugs were administered to all patients and no prophylactic antibiotics were administered. All patients were routinely admitted postSOVP for 48 hours.

The endpoints of the study were technical success, clinical success and adverse event (AE) rates. Overall technical success was defined by achievement of two subitems: SOVP success and PD clearance success. SOVP success was defined as the ability to reach the target stone with SpyGlassDS. PD clearance success was defined as PD decompression by complete PD stone clearance or significant duct clearance making stent placement possible.

Clinical success was defined as a $>50 \%$ post-interventional reduction on a numeric rating scale (NRS) for pain. The NRS is a segmented numeric version of the visual analog scale (VAS) in which a whole number $(0-10)$ that best reflects the intensity of their pain is chosen [8].

A systematic medical record review comprising clinical evaluation and biochemistry was used to determine the occurrence of AEs [4]. AE rates and severity of AEs were reported according to the American Society for Gastrointestinal Endoscopy guidelines, therefore PEP was classified by the Cotton criteria [9].

Patients were rescheduled 3 to 6 months after SOVP for stent extraction and were also interviewed for clinical success and quality of life using a standardized questionnaire ( $\triangleright$ Table 1). Statistical significance was tested with two-tailed $t$-test or fisher's exact test. A $P<0.05$ was considered statistically significant. GraphPad prism 5, MS Excel and medcalc.org were 
- Table 1 Systematic questionnaire for follow-up (in parts adjusted from UK SF-12 Score - Jenkinson et al. Journal of Puplic health medicine 1997).

\begin{tabular}{l|l|}
$\begin{array}{l}\text { 1) How would you describe your health status in } \\
\text { general? }\end{array}$ & $\begin{array}{l}1 \text { excellent } \\
2 \text { very good } \\
3 \text { good } \\
4 \text { fair } \\
5 \text { poor }\end{array}$ \\
\hline $\begin{array}{l}\text { 2) How much did pain affect you in in your daily } \\
\text { life and work? }\end{array}$ & $\begin{array}{l}1 \text { not at all } \\
2 \text { a little bit } \\
3 \text { moderately } \\
4 \text { very much }\end{array}$ \\
\hline $\begin{array}{l}\text { 3) In a numerical range of } 1-10 \text { of which } 10 \text { is the } \\
\text { worst imaginable pain: How would you describe } \\
\text { your pain level at the moment? }\end{array}$ & $1-10$ \\
\hline $\begin{array}{l}\text { 4) The interventional procedure has reduced my } \\
\text { symptoms }\end{array}$ & Yes/No \\
\hline $\begin{array}{l}\text { 5) Since the intervention my daily use of painkillers } \\
\text { is reduced noticeable }\end{array}$ & Yes/No \\
\hline
\end{tabular}

used as statistical tools. The study had medical ethics committee approval and written consent was obtained from all participating patients.

\section{Results}

A total of 23 interventions in 20 patients ( $\$$ Table 2 ) were performed. Prior unsuccessful interventional procedures on pancreatic calculi or strictures had been performed in $95 \%$ of the cases ( $>$ Table 2 ).

Eight of 20 patients had a PD stenosis downstream to the stone(s) and all patients had an upstream PD dilation $>5 \mathrm{~mm}$.

SOVP success rate was achieved in $100 \%$ of cases and PD clearance success was achieved in 19 of 20 patients (95\%). Overall technical success rate was $95 \%$. The Holmium laser was used in 21 interventions and EHL in 2. All EHL lithotripsies were successful. Stone fragments could be completely removed with endoscopic and fluoroscopic visualization in 13 cases (68\%), whereas in six cases (32\%) the stone could be sufficiently reduced in size to allow for PD decompression and stent placement, which had not been possible before. In 19 of 20 patients a plastic stent (5FR-10FR) was placed due to a persistent stricture or for PEP prophylaxis. In two cases, remaining calculi could not be detected 3 months after POP-guided lithotripsy, when patients underwent a subsequent ERCP for stent extraction. In another two cases calculi could be extracted in a subsequent intervention with standard ERCP methods. Prophylactic placed stents (5FR) could be removed at next ERCP. All eight patients with strictures still needed stenting at 6-month follow-up. Three patients needed a stent at 3-month follow-up due to persistent dilatation, but these stents could be extracted at 6-month follow-up. All other patients (9 of 12; $75 \%$ ) had no stents after 3-month follow-up.
- Table 2 Patient data and characteristics of stone and main pancreatic duct.

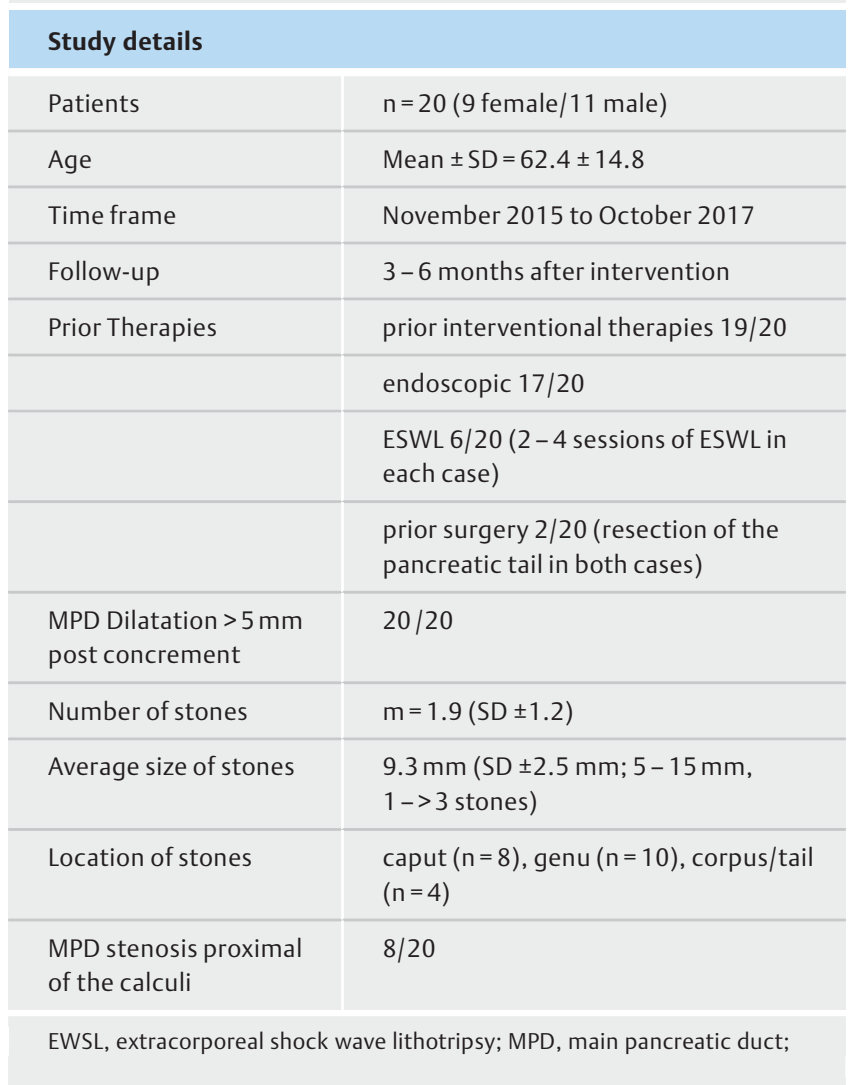

Stone location, number of stones and presence of downstream PD stenosis did not significantly influence success rates ( $\triangleright$ Table 2 ). AEs occurred in seven of 23 procedures (30\%). In one case, contrast extravasation from the PD was fluoroscopically identified and could be treated by temporary plastic stenting in the same session. This was rated as a serious AE. The patient also developed a moderate PEP. PEP occurred in five other procedures (total PEP rate $26 \%$ ) of which four were classified as mild and two as moderate (Cotton criteria). PEP occurred significantly more frequently in patients with prior PEP $(n=4$, $P<0.01)$. Post-sphincterotomy bleeding occurred in one patient and was self-limiting.

Patients reported an improvement of pain 24 to 48 hours after treatment. At 3- to 6-months follow-up, $95 \%$ of patients reported improvement in symptoms and reduction in intake of analgesics. One patient was lost to follow-up. Mean NRS decreased from $5.4[ \pm 1.6]$ to $2.8[ \pm 1.8](P<0.01)$. Clinical success was achieved in $95 \%$ of patients. Concerning post-interventional quality of life, $89 \%$ reported no or only mild disability in daily activities and $47 \%$ described their general health status as "excellent" or "very good" afterwards. 


\section{Discussion}

We report a high technical success rate and clinical success rate of $95 \%$, respectively and an acceptable $\mathrm{AE}$ rate of $30 \%$, despite the fact that we included a challenging patient population with prior unsuccessful therapies and large PD stones. Forty percent of patients had strictures that could be successfully treated endoscopically in the same session, suggesting that strictures are not an impediment to technical success.

ESWL as a standard of care has a reported success rate of $59 \%$ to $80 \%$ after an average of three sessions and an AE rate of $5.8 \%$ to $6.7 \%$ [10 - 13]. Our data suggest a potentially higher success rate of $95 \%$, but with a higher AE rate of $30 \%$ and occurrence of PEP of $26 \%$. Due to the small cohort, no definite answer can be provided as to whether laser lithotripsy or EHL is more effective. However, it is in line with reported PEP rates in average high-risk ERCP patients of $20.2 \%$ to $40 \%$ [ $14-15$ ] and in SOVP patients with PEP up to $28 \%[2,4,6]$. Risk factors include patient-, physician- or procedure-related and are additive [14]. Many of those factors were consistently present in our patients (e.g. prior PEP, difficult cannulation, pancreatic sphincterotomy, pancreatic duct injection). Even in our small patient cohort, a prior episode of PEP was identified as a risk factor for PEP following SOVP ( $P=0.01$ in Fisher's exact test, OR 25.0, 95\%-Cl: 1.11 to $562.8, P=0.043)$. Therefore, careful patient selection and an intensified PEP prophylaxis scheme, with rectal NSAIDS and possibly PD stenting, is warranted in case of SOVP.

To our knowledge, this is the largest cohort of patients that have been evaluated, focusing on PD stones only with digital SOVP (SpyGlassDS). Strengths of our study include a high technical and clinical success rate, structured follow-up and a technically difficult patient population that already had failed previous treatments. Limitations of our study include its retrospective, uncontrolled design, lack of an intention-to-treat analysis, short-term follow-up and modest number of patients. Therefore, our results need to be interpreted cautiously given that this was a retrospective pilot study designed primarily to assess feasibility and safety of SOVP to treat PD stones in obstructing CCP, and to generate information for design of future trials. Looking at the relatively high technical and clinical success rates, we cannot rule out the possibility that the significant benefit of SOVP reflects an overestimation of the effect size, due to the small sample size.

Initial endoscopic therapy (including ESWL) is likely to be inferior to surgery for treatment of obstructing CCP, though a definite answer will be given by the results of the ESCAPE trial. Therefore, use of SOVP in patients with obstructive CCP due to PD stones is limited to a small group of patients, i. e. poor surgical candidates and/or patients who refuse surgery. Direct cost and resource comparisons are not possible due to the retrospective design and relatively small sample size and require assessment through prospectively designed trials. The clinical outcome regarding quality of life and pain suggests a significant improvement in the vast majority. It remains to be seen if these results from our small cohort can match the long-term pain relief after ESWL in $60 \%$ to $90 \%$ of the cases [10].

\section{Conclusion}

In conclusion, SOVP-guided lithotripsy was shown to be safe and effective also in patients with prior unsuccessful interventional therapy. Complete or partial stone removal had a significant beneficial effect on pain reduction and quality of life. Prospective studies are required to validate our findings and to help establish the role of SOVP in treatment of symptomatic PD stones in CCP. At this stage, this treatment cannot be recommended as a first-line approach for fragmentation of stones in CCP, due to its technical difficulty and the availability of less invasive alternatives, such as ESWL.

\section{Competing interests}

None

References

[1] Hoffmeister A. Chronic Pancreatitis German Society of D, Metabolic D. et al. S3-Consensus guidelines on definition, etiology, diagnosis and medical, endoscopic and surgical management of chronic pancreatitis German Society of Digestive and Metabolic Diseases (DGVS). Z Gastroenterol 2012; 50: 1176-1224

[2] Attwell AR, Patel S, Kahaleh M et al. ERCP with per-oral pancreatoscopy-guided laser lithotripsy for calcific chronic pancreatitis: a multicenter U.S. experience. Gastrointest Endosc 2015; 82: 311-318

[3] Attwell AR, Brauer BC, Chen YK et al. Endoscopic retrograde cholangiopancreatography with per oral pancreatoscopy for calcific chronic pancreatitis using endoscope and catheter-based pancreatoscopes: a 10-year single-center experience. Pancreas 2014; 43: 268-274

[4] Beyna T, Neuhaus H, Gerges C. Endoscopic treatment of pancreatic duct stones under direct vision: Revolution or resignation? Systematic review Dig Endosc 2018; 30: 29-37

[5] Lohr JM, Dominguez-Munoz E, Rosendahl J et al. United European Gastroenterology evidence-based guidelines for the diagnosis and therapy of chronic pancreatitis (HaPanEU). United European Gastroenterol J 2017; 5: 153-199

[6] Bekkali NL, Murray S, Johnson G] et al. Pancreatoscopy-directed electrohydraulic lithotripsy for pancreatic ductal stones in painful chronic pancreatitis using SpyGlass. Pancreas 2017; 46: 528- 530

[7] Maydeo A, Kwek BE, Bhandari S et al. Single-operator cholangioscopyguided laser lithotripsy in patients with difficult biliary and pancreatic ductal stones (with videos). Gastrointest Endosc 2011; 74: 1308 1314

[8] Hawker GA, Mian S, Kendzerska T et al. Measures of adult pain: Visual Analog Scale for Pain (VAS Pain), Numeric Rating Scale for Pain (NRS Pain), McGill Pain Questionnaire (MPQ), Short-Form McGill Pain Questionnaire (SF-MPQ), Chronic Pain Grade Scale (CPGS), Short Form-36 Bodily Pain Scale (SF-36 BPS), and Measure of Intermittent and Constant Osteoarthritis Pain (ICOAP). Arthritis Care Res (Hoboken) 2011; 63: S240-252

[9] Cotton PB, Lehman G, Vennes J et al. Endoscopic sphincterotomy complications and their management: an attempt at consensus. Gastrointest Endosc 1991; 37: 383-393

[10] Tandan M, Talukdar R, Reddy DN. Management of pancreatic calculi: an update. Gut Liver 2016; 10: $873-880$

[11] Dumonceau JM, Costamagna G, Tringali A et al. Treatment for painful calcified chronic pancreatitis: extracorporeal shock wave lithotripsy 
versus endoscopic treatment: a randomised controlled trial. Gut 2007; 56: $545-552$

[12] Dumonceau JM, Delhaye M, Tringali A et al. Endoscopic treatment of chronic pancreatitis: European Society of Gastrointestinal Endoscopy (ESGE) Clinical Guideline. Endoscopy 2012; 44: 784-800

[13] Li BR, Liao Z, Du TT et al. Risk factors for complications of pancreatic extracorporeal shock wave lithotripsy. Endoscopy 2014; 46: 1092 1100
[14] Thaker AM, Mosko JD, Berzin TM. Post-endoscopic retrograde cholangiopancreatography pancreatitis. Gastroenterol Rep (Oxf) 2015; 3: $32-40$

[15] Elmunzer BJ, Scheiman JM, Lehman GA et al. A randomized trial of rectal indomethacin to prevent post-ERCP pancreatitis. N Engl J Med 2012; 366: $1414-1422$ 